\title{
Optimal control problem for semilinear parabolic equations with pointwise state constraints
}

\author{
J. P. Raymond \\ Université Paul Sabatier, UMR CNRS MIP \\ 31062 Toulouse Cedex France, Fax: (33) 61558385. \\ Phone: (33) 615583 14. E-mail: raymond@cict.fr
}

\begin{abstract}
This paper deals with optimal control problems governed by semilinear parabolic equations in presence of pointwise constraints on the state variable. We obtain necessary optimality conditions in which the adjoint state satisfies a parabolic equation with measures as source terms. We prove new existence and regularity results for solutions of such equations. For simplicity we only consider the case of a boundary control, but the results can be extended to problems with a control in the initial condition and a distributed control.
\end{abstract}

\section{Keywords}

Nonlinear boundary controls, semilinear parabolic equations, pointwise state constraints

\section{THE CONTROL PROBLEM}

We consider the following state equation

$$
\begin{aligned}
& \left.\frac{\partial y}{\partial t}+A y+\Phi(x, t, y)=0 \quad \text { in } \quad Q=\Omega \times\right] 0, T[, \\
& \left.\frac{\partial y}{\partial n_{A}}+\Psi(y, v)=0 \quad \text { on } \Sigma=\partial \Omega \times\right] 0, T[ \\
& y(x, 0)=y_{0}(x) \text { in } \Omega,
\end{aligned}
$$

$\Omega$ is a bounded regular subset of $R^{N}, A$ is a second order symmetric uniformly elliptic operator with regular coefficients $\left(A y=-\Sigma_{i, j} D_{j}\left(a_{i j}(x) D_{i} y\right)\right), y_{0}$ belongs to $C(\bar{\Omega})$, assumptions on $\Phi$ and $\Psi$ are given below, the control $v$ satisfies

$v \in K_{V}$

$K_{V}$ is a closed convex subset of $L^{\beta r}(\Sigma)$ (for $r>N+1$ and some $\beta \geq 1$ ). The pointwise constraints are of the form

$g(x, t, y(x, t)) \leq 0 \quad$ for every $(x, t) \in \bar{Q}$. 
We suppose that $y_{0}$ is such that $g\left(x, 0, y_{0}(x)\right) \leq 0$ on $\bar{\Omega}$. These constraints are meaningful since we prove in (Raymond and Zidani, 1995) that the solution of (1) belongs to $C(\bar{Q})$ if assumptions (A1) and (A2) (given below) are satisfied. The cost functional is defined by

$J(y, v)=\int_{Q} F(x, t, y) d x d t+\int_{\Sigma} G(y, v) d s d t+\int_{\Omega} H(x, y(x, T)) d x$

and the control problem by

$\inf \{J(y, v) \mid(y, v)$ satisfies $(1)-(3)\}$.

In all the sequel, we make the following assumptions.

(A1) For every $y \in R, F(., y)$ and $\Phi(., y)$ are measurable on $Q$. For almost every $(x, t) \in$ $Q, F(., y)$ and $\Phi(., y)$ are of class $C^{1}$ and we have the following estimates

$|\Phi(x, t, y)|+|F(x, t, y)|+\left|F_{y}^{\prime}(x, t, y)\right| \leq \eta(|y|), \quad c_{0} \leq \Phi_{y}^{\prime}(x, t, y) \leq \eta(|y|)$,

where $\eta$ is a nondecreasing function from $R^{+}$into $R^{+}$and $c_{0} \in R$. (We have denoted by $F_{y}^{\prime}$ and $\Phi_{y}^{\prime}$ the partial derivatives of $F$ and $\Phi$ with respect to $y$, in all the sequel we adopt the same kind of notation for other functions).

(A2) $\Psi$ and $G$ are continuous on $R^{2}$, for every $v \in R, \Psi(., v)$ and $G(., v)$ are of class $C^{1}$ on $R$ and we have the following estimates

$\left|G_{y}^{\prime}(y, v)\right|+|G(y, v)| \leq\left(1+|v|^{\beta r}\right) \eta(|y|), \quad\left|G_{v}^{\prime}(y, v)\right| \leq\left(1+|v|^{\beta r-1}\right) \eta(|y|)$,

$c_{0} \leq \Psi_{y}^{\prime}(y, v) \leq\left(1+|v|^{\beta}\right) \eta(|y|), \quad|\Psi(y, v)| \leq\left(1+|v|^{\beta}\right) \eta(|y|)$,

$\left|\Psi_{v}^{\prime}(y, v)\right| \leq\left(1+|v|^{\beta-1}\right) \eta(|y|)$,

where $\eta$ is as in (A1).

(A3) For every $x \in \Omega, H(x,$.$) is of class C^{1}$ on $R$, for every $y \in R, H(., y)$ is measurable on $\Omega$,

$\left|H_{y}^{\prime}(x, y)\right|+|H(x, y)| \leq \eta(|y|)$.

The function $g$ is continuous on $\bar{Q} \times R$ and, for every $(x, t)$ in $\bar{Q}, g(x, t,$.$) is of$ class $C^{1}$ on $R$.

Under additional assumptions, we have proved that $(P)$ admits solutions (Raymond, 1995). Here, we are mainly interested in optimality conditions.

\section{PARABOLIC EQUATIONS WITH MEASURES}

Since the adjoint equation in optimality conditions for $(P)$ is a parabolic equation with measures as data, we first consider such equations for which we prove existence and regularity results.

For every $(a, b) \in L^{\infty}(Q) \times L^{r}(\Sigma)$, we consider the following terminal boundary value problem: 
$-\frac{\partial p}{\partial t}+A p+a p=\mu_{Q}$ in $Q$,

$\frac{\partial p}{\partial n_{A}}+b p=\mu_{\Sigma}$ on $\Sigma$,

$p(T)=\mu_{\bar{\Omega}_{T}}$ in $\bar{\Omega}$,

where $\mu=\mu_{Q}+\mu_{\Sigma}+\mu_{\Omega_{T}}$ is a bounded Radon measure on $\bar{Q} \backslash \bar{\Omega} \times\{0\}, \mu_{Q}$ is the restriction of $\mu$ to $Q, \mu_{\Sigma}$ is the restriction of $\mu$ to $\Sigma$ and $\mu_{\Omega_{T}}$ is the restriction of $\mu$ to $\bar{\Omega} \times\{T\}$.

By definition a function $p \in L^{1}\left(0, T ; W^{1,1}(\Omega)\right)$ is a weak solution of (4) if $b p \in L^{1}(\Sigma)$ and if

$\int_{Q}\left(p \frac{\partial y}{\partial t}+\sum_{i, j} a_{i j} D_{j} p D_{i} y+a p y\right) d x d t+\int_{\Sigma} b p y d s d t=\langle y, \mu\rangle_{C_{b}\left(\bar{Q} \mid \bar{\Omega}_{0}\right) \times \mathcal{M}_{b}\left(\bar{Q} \mid \bar{\Omega}_{0}\right)}$

for every $y \in C^{1}(\bar{Q})$ satisfying $y(x, 0)=0$ in $\bar{\Omega}$.

Thanks to some new regularity results on linear parabolic equations (see Proposition 2) and thanks to duality arguments we can prove the following result (Raymond, 1995).

Theorem 1 There exists a unique weak solution $p$ of (4) in $L^{1}\left(0, T ; W^{1,1}(\Omega)\right)$, for every $(\delta, d)$ satisfying $\delta>1, d>1, \frac{d}{\delta^{\prime}}-\frac{d}{2}>\frac{N}{2}, \delta \leq r, \frac{N r}{r-2}<d \leq \frac{N r}{N-1}, p$ belongs to $L^{\delta^{\prime}}\left(0, T ; W^{1, d^{\prime}}(\Omega)\right)$. The normal trace on $\partial Q$ of the vector field $\left(\left(\sum_{j} a_{i j} D_{j} p\right)_{1 \leq i \leq N}, p\right)$ belongs to $\mathcal{M}(\partial Q)$ (the space of Radon measure on $\partial Q$ ) and $p$ verifies the following Green formula

$$
\begin{aligned}
& \int_{Q} p\left(\frac{\partial y}{\partial t}+A y+a y\right) d x d t+\int_{\Sigma} p\left(\frac{\partial y}{\partial n_{A}}+b y\right) d s d t= \\
& \left\langle y,-\frac{\partial p}{\partial t}+A p+a p\right\rangle_{C_{b}(Q) \times \mathcal{M}_{b}(Q)}+\left\langle y, \frac{\partial p}{\partial n_{A}}+b p\right\rangle_{C_{b}(\Sigma) \times \mathcal{M}_{b}(\Sigma)}+ \\
& +\langle y(T), p(T)\rangle_{C(\bar{\Omega} \times\{T\}) \times \mathcal{M}(\bar{\Omega} \times\{T\})}-\langle y(0), p(0)\rangle_{C(\bar{\Omega} \times\{0\}) \times \mathcal{M}(\bar{\Omega} \times\{0\})},
\end{aligned}
$$

where $p(0)$ is the restriction to $\bar{\Omega} \times\{0\}$ of the normal trace of $\left(-\left(\sum_{j} a_{i j} D_{j} p\right)_{1 \leq i \leq N},-p\right)$.

Remark The conditions $\delta \leq r$ and $\frac{N r}{r-2}<d \leq \frac{N r}{N-1}$ can be omitted if $b \in L^{\infty}(\Sigma)$. Since the divergence in the sense of distributions in $Q$ of the vector field $\left(\left(\sum_{j} a_{i j} D_{j} p\right)_{1 \leq i \leq N}, p\right)$ is a bounded Radon measure in $Q$, we can define the normal trace on $\partial Q$ of this vector field in some Sobolev space with a negative exponent which contains the space of Radon measures on $\partial Q$ (see Casas, 1993).

To prove Theorem 1 we proceed as in (Alibert and Raymond, 1994) where the case of elliptic equations is treated. We consider sequences of regular functions $\left(h_{n}\right)_{n} \subset C_{c}(Q)$ (the space of continuous functions with compact support in $Q),\left(k_{n}\right)_{n} \subset C_{c}(\Sigma)$ and $\left(\ell_{n}\right)_{n} \subset$ $C(\bar{\Omega})$ such that $\left(k_{n}\right)_{n}$ converges to $\mu_{Q}$ for the narrow topology of $\mathcal{M}_{b}(Q),\left(k_{n}\right)_{n}$ converges to $\mu_{\Sigma}$ for the narrow topology of $\mathcal{M}_{b}(\Sigma)$ and $\left(\ell_{n}\right)_{n}$ converges to $\mu_{\bar{\Omega}_{T}}$ for the weak star topology of $\mathcal{M}(\bar{\Omega})$. We denote by $\left(p_{n}\right)_{n}$ the solution of

$-\frac{\partial p}{\partial t}+A p+a p=h_{n} \quad$ in $Q, \quad \frac{\partial p}{\partial n_{A}}+b p=k_{n} \quad$ on $\Sigma, \quad p(T)=\ell_{n} \quad$ in $\Omega$. 
The main point in the proof of Theorem 1 is the following estimate

$$
\left\|p_{n}\right\|_{L^{\delta^{\prime}\left(0, T ; W^{1, d^{\prime}}(\Omega)\right)}} \leq C\left(\left\|h_{n}\right\|_{L^{1}(Q)}+\left\|k_{n}\right\|_{L^{1}(\Sigma)}+\left\|\ell_{n}\right\|_{L^{1}(\Omega)}\right)
$$

with $C$ independent of $n$. To prove such a result, we proceed by duality, we denote by $y$ the solution of

$$
\frac{\partial y}{\partial t}+A y+a y=f-\operatorname{div} \xi \quad \text { in } Q, \quad \frac{\partial y}{\partial n_{A}}+b y=0 \quad \text { on } \Sigma, \quad y(0)=0 \quad \text { in } \Omega,
$$

where $(f, \xi) \in \mathcal{D}(Q) \times \mathcal{D}\left(Q ; R^{N}\right)$. With a Green formula we get

$$
\left|\int_{Q}\left(f p_{n}+\xi \cdot D p_{n}\right) d x d t\right|=\left|\int_{Q} h_{n} y+\int_{\Sigma} k_{n} y+\int_{\Omega} \ell_{n} y\right| \text {. }
$$

Thanks to (8) and to Proposition 2 (see below), we can finally prove (6) and we can pass to the limit in the weak formulation of the equation satisfied by $p_{n}$. The Green formula of Theorem 1 is also obtained by a passage to the limit. The main difficulty is to correctly justify the writing $\langle y(0), p(0)\rangle_{C(\bar{\Omega} \times\{0\}) \times \mathcal{M}(\bar{\Omega} \times\{0\})}$.

Proposition 2 For every $a \in L^{\infty}(Q)$, every $b \in L^{r}(\Sigma)$ satisfying $a \geq c_{0}, b \geq c_{0}$ for some $c_{0} \in R$, and for every $(f, \xi) \in \mathcal{D}(Q) \times \mathcal{D}\left(Q ; R^{N}\right)$, there exists a unique weak solution $y$ of (7) in $L^{2}\left(0, T ; H^{1}(\Omega)\right) \cap C\left([0, T] ; L^{2}(\Omega)\right)$. This solution belongs to $C(\bar{Q})$ and satisfies the estimate

$\|y\|_{L^{2}\left(0, T ; H^{1}(\Omega)\right)}+\|y\|_{C(\bar{Q})} \leq C\left(\|f\|_{L^{\nu}\left(0, T ; L^{m}(\Omega)\right)}+\sum_{i=1}^{N}\left\|\xi_{i}\right\|_{L^{\delta}\left(0, T ; L^{d}(\Omega)\right)}\right)$,

for any $(\nu, m, \delta, d)$ such that

$m>1, \quad \nu>1, m / \nu^{\prime}>N / 2$,

$\delta>1, \quad d>1, \quad \frac{d}{\delta^{\prime}}-\frac{d}{2}>\frac{N}{2}$,

and the constant $C=C\left(c_{0}, \nu, m, r, \delta, d, \Omega, T\right)$ does not depend on $a, b, f$ and $\xi$.

Estimate in Proposition 2 is a classical result for parabolic equations with homogeneous Dirichlet boundary conditions (Lady ženskaya, Solonnikov and Ural'ceva, 1968). Here we cannot use these results because we deal with Robin boundary conditions. Moreover, since $b$ (the coefficient in the boundary condition) can be negative, we cannot use a truncation method to get $L^{\infty}$-estimates as in (Ladyżenskaya, Solonnikov and Ural'ceva, 1968, Chapter 3). To prove Proposition 2, we first consider the solution $\tilde{y}$ of

$\frac{\partial y}{\partial t}+A y+c_{1} y=e^{-\left(c_{1}-c_{0}\right) t}(f-\operatorname{div} \xi)$ in $Q$,

$\frac{\partial y}{\partial n_{A}}+c_{0} y=0 \quad$ on $\Sigma, \quad y(0)=0 \quad$ in $\Omega$

where $c_{1}$ is a constant sufficiently big. Still by using duality arguments and estimates on analytic semigroups, we can prove the following estimate for $\tilde{y}$ 
$\|\tilde{y}\|_{C(\bar{Q})} \leq C\left(\|f\|_{L^{\nu}\left(0, T ; L^{m}(\Omega)\right)}+\sum_{i=1}^{N}\left\|\xi_{i}\right\|_{L^{\delta}\left(0, T ; L^{d}(\Omega)\right)}\right)$.

Thanks to this estimate and to a new comparison principle (Raymond and Zidani, 1995), we obtain

$0 \leq e^{-\left(c_{1}-c_{0}\right) t} y^{+} \leq \tilde{y}^{+}$

where $y^{+}=\operatorname{Max}(y, 0)$ and $\tilde{y}^{+}=\operatorname{Max}(\tilde{y}, 0)$. We get a similar estimate for $y^{-}$and we can conclude. The comparison principle given in (Raymond and Zidani, 1995) extends a result proved in (Schmidt, 1989) to the case when $b$ is not bounded (only bounded from below) and can be negative.

\section{OPTIMALITY CONDITIONS}

We now give an existence theorem of Lagrange multipliers for control problems proved in (Alibert and Raymond 1994). We use the following notation

$Z_{0}=Y \times \Pi \quad$ and $\quad C_{0}=Y \times \Pi_{a d}$,

where $Y$ and $\Pi$ are Banach spaces, $\Pi$ is separable, $\Pi_{a d}$ is a nonempty closed convex subset of $\Pi$. For $i=1,2, Z_{i}$ is a Banach space, $Z_{i}^{*}$ its topological dual, $G_{1}$ is a mapping from $Z_{0}$ into $Z_{1}, G_{2}$ is a mapping from $Y$ into $Z_{2}, J$ is a functional defined on $Z_{0}$ and we denote by $C_{2}$ a convex cone in $Z_{2}$ with nonempty interior and vertex at the origin. We consider the following abstract control problem.

$\inf \left\{J(y, \pi) \mid(y, \pi) \in C_{0}, G_{1}(y, \pi)=0, G_{2}(y) \in C_{2}\right\}$.

Theorem 3 Let $(\bar{y}, \bar{\pi})$ be an optimal point for $(C P)$ let us assume that

(i) $J$ is Fréchet-differentiable at $(\bar{y}, \bar{\pi})$ and $G_{2}$ is Fréchet-differentiable at $\bar{y}$,

(ii) $G_{1}$ is strictly differentiable at $(\bar{y}, \bar{\pi})$ and the linear operator $G_{1 y}^{\prime}(\bar{y}, \bar{\pi})$ from $Y$ into $Z_{1}$ is surjective,

(iii) there exists $y_{0} \in Y$ such that

$$
G_{2}(\bar{y})+G_{2}^{\prime}(\bar{y}) y_{0} \in \text { int } C_{2} .
$$

There then exists $(p, \mu, \lambda) \in Z_{1}^{*} \times Z_{2}^{*} \times R$, with $(\mu, \lambda) \neq(0,0)$, satisfying

$\lambda J_{y}^{\prime}(\bar{y}, \bar{\pi})+p G_{1 y}^{\prime}(\bar{y}, \bar{\pi})+\mu G_{2}^{\prime}(\bar{y})=0$,

$\lambda J_{\pi}^{\prime}(\bar{y}, \bar{\pi})(\pi-\bar{\pi})+\left\langle p, G_{1 \pi}^{\prime}(\bar{y}, \bar{\pi})(\pi-\bar{\pi})\right\rangle_{Z_{1}^{*} \times Z_{1}} \geq 0$,

for every $\pi \in \Pi_{a d}$,

$\lambda \geq 0, \mu \in C_{2}^{*}$ and $\left\langle\mu, G_{2}(\bar{y})\right\rangle_{Z_{2}^{*} \times Z_{2}}=0$, 
$\left(C_{2}^{*}\right.$ is the polar cone of $\left.C_{2}\right)$. If moreover there exist $\pi_{0} \in \Pi_{a d}$ and $y_{0} \in Y$ such that

$G_{1 y}^{\prime}(\bar{y}, \bar{\pi}) y_{0}+G_{1 \pi}^{\prime}(\bar{y}, \bar{\pi}) \pi_{0}=0 \quad$ and $\quad G_{2}(\bar{y})+G_{2}^{\prime}(\bar{y}) y_{0} \in$ int $C_{2}$

then we can take $\lambda=1$ in (11) and (12).

We denote by $Y$ the space

$Y=\left\{y \in W\left(0, T ; H^{1}(\Omega),\left(H^{1}(\Omega)\right)^{\prime}\right) \mid \frac{\partial y}{\partial t}+A y \in L^{\infty}(Q), \frac{\partial y}{\partial n_{A}} \in L^{r}(\Sigma), y(0) \in C(\bar{\Omega})\right\}$

endowed with the graph norm.

Let $(\bar{y}, \bar{v}) \in Y \times K_{V}$ be a solution of the optimal control problem $(P)$. We can apply Theorem 3 to $(P)$. For this, we set:

$\Pi=L^{\beta r}(\Sigma), \quad Z_{0}=Y \times \Pi, \quad C_{0}=Y \times \Pi_{a d}, \quad \Pi_{a d}=K_{V}$,

$Z_{1}=L^{\infty}(Q) \times L^{r}(\Sigma) \times C(\bar{\Omega}), \quad Z_{2}=C(\bar{Q})$,

$C_{2}=\{h \in C(\bar{Q}) \mid h(x, t) \leq 0$ for all $(x, t) \in \bar{Q}\}$,

$G_{1}(y, v)=\left(\frac{\partial y}{\partial t}+A y+\Phi(., y), \frac{\partial y}{\partial n_{A}}+\Psi(y, v), y(0)-y_{0}\right)$

and $\quad G_{2}(y)=g(., y)$.

The surjectivity of $G_{1 y}^{\prime}(\bar{y}, \bar{v})$ has been proved in (Raymond and Zidani, 1995).

We shall say that $(\bar{y}, \bar{v}) \in C(\bar{Q}) \times K_{V}$ satisfies the weak qualification condition for $(P)$ if there exists $z \in C(\bar{Q})$ satisfying

$g(x, t, \bar{y}(x, t))+g_{y}(x, t, \bar{y}(x, t)) z(x, t)<0 \quad$ on $\quad \bar{Q}$.

Since $Y$ is dense in $C(\bar{Q})$, this qualification condition corresponds to condition (10) of Theorem 3.

We shall say that $(\bar{y}, \bar{v}) \in C(\bar{Q}) \times K_{V}$ satisfies the strong qualification condition for $(P)$ if there exists $(z, v) \in C(\bar{Q}) \times K_{V}$ satisfying

$g(., \bar{y})+g_{y}(., \bar{y}) z<0 \quad$ on $\bar{Q}$,

$\frac{\partial z}{\partial t}+A z+\Phi_{y}^{\prime}(., \bar{y}) z=0$ in $Q, \quad \frac{\partial z}{\partial n_{A}}+\Psi_{y}^{\prime}(\bar{y}, \bar{v}) z+\Psi_{v}^{\prime}(\bar{y}, \bar{v}) v=0$ on $\Sigma, \quad z(0)=0 \quad$ in $\Omega$.

The strong qualification condition corresponds to condition (13) of Theorem 3.

Thanks to Theorem 1 and Theorem 3 , we can finally prove the following result.

Theorem 4 If $(\bar{y}, \bar{v})$ is a solution of $(P)$ and if $(\bar{y}, \bar{v})$ satisfies the weak qualification. condition for $(P)$, there then exist $\lambda \in R^{+}, p \in L^{\delta^{\prime}}\left(0, T ; W^{1, d^{\prime}}(\Omega)\right)$ for all $(\delta, d)$ satisfying $\delta>1, d>1, \frac{d}{\delta^{\prime}}-\frac{d}{2}>\frac{N}{2}, \delta \leq r, \frac{N r}{r-2}<d \leq \frac{N r}{N-1}$, and $\mu \in \mathcal{M}_{b}(\bar{Q} \backslash \bar{\Omega} \times\{0\})$ (the space of bounded Radon measures on $\bar{Q} \backslash \bar{\Omega} \times\{0\}$ ) such that

$-\frac{\partial p}{\partial t}+A p+\Phi_{y}^{\prime}(., \bar{y}) p+\lambda F_{y}^{\prime}(., \bar{y})+\mu_{Q} g_{y}^{\prime}(., \bar{y})=0$ in $Q$, 


$$
\begin{aligned}
& \frac{\partial p}{\partial n_{A}}+\Psi_{y}^{\prime}(\bar{y}, \bar{v}) p+\lambda G_{y}^{\prime}(\bar{y}, \bar{v})+\mu_{\Sigma} g_{y}^{\prime}(., \bar{y})=0 \text { on } \Sigma, \\
& p(T)=-\lambda H_{y}^{\prime}(., \bar{y}(T))-\mu_{\Omega_{T}} g_{y}^{\prime}(., T, y(T)) \text { on } \bar{\Omega}, \\
& \int_{\Sigma}\left(\lambda G_{v}^{\prime}(\bar{y}, \bar{v})+p \Psi_{v}^{\prime}(\bar{y}, \bar{v})\right)(v-\bar{v})(s, t) d s d t \geq 0 \text { for all } v \in K_{V}, \\
& (\lambda, \mu) \not \equiv 0, \mu \geq 0,\langle\mu, g(., \bar{y})\rangle_{\mathcal{M}_{b}(\bar{Q} \backslash \bar{\Omega} \times\{0\}) \times C_{b}(\bar{Q} \backslash \bar{\Omega} \times\{0\})}=0,
\end{aligned}
$$

where $\mu_{Q}$ is the restriction of $\mu$ to $Q, \mu_{\Sigma}$ is the restriction of $\mu$ to $\Sigma$ and $\mu_{\Omega_{T}}$ is the restriction of $\mu$ to $\bar{\Omega} \times\{T\}$ (Equation (14) is satisfied in the sense of definition given in (5)). If moreover $(\bar{y}, \bar{v})$ satisfies the strong qualification condition, we can take $\lambda=1$ in (14)-(16).

These results extend to problems governed by semilinear parabolic equations previous results obtained when the state equation is elliptic (see Casas (1993), and Alibert and Raymond (1994) where the case of nonlinear boundary conditions and equations with non regular coefficients is considered). The regularity results for equation (1) have been obtained with H. Zidani (Raymond and Zidani (1995)). Existence of a solution in $L^{1}(Q)$ for equation (4) is obtained in (Mackenroth, 1982) when $a \equiv 0$ and $b$ is a positive constant. Let us stress on that here $b$ can be negative. Optimality conditions are also obtained in (Fattorini and Murthy, 1994) for problems with terminal state constraints.

\section{REFERENCES}

Alibert, J. J. and Raymond, J. P. (1994), Optimal control problems governed by semilinear elliptic equations with pointwise state constraints, submitted to SIAM J. Cont. Optim..

Casas, E. (1993) Boundary Control of Semilinear Elliptic Equations with Pointwise State Constraints, SIAM J. Control Optim., 31, 993-1006.

Fattorini, H. O. and Murthy, T. (1994), Optimal problems for nonlinear parabolic boundary control systems, SIAM J. Cont. Opt., 32, 1577-1596.

Ladyženskaya, O. A., Solonnikov, V. A. and Ural'ceva, N.N. (1968) Linear and quasilinear equations of parabolic type, AMS Translations of Mathematical Monographs 23, Providence.

Mackenroth, U. (1982) Convex Parabolic Boundary Control Problems with Pointwise State Constraints, J. Math. Anal. Appli., 87, 256-277.

Schmidt, E.J. (1989) Boundary Control for the Heat Equation with Nonlinear Boundary Condition, J. Diff. Equat., 78, 89-121.

Raymond, J.P. and Zidani, H. (1995) Hamiltonian Pontryagin's Principles for Control Problems Governed by Semilinear Parabolic Equations, preprint.

Raymond, J.P. (1995) Nonlinear boundary control of semilinear parabolic equations with pointwise state constraints, preprint. 